\title{
A Multi Criteria Meta-heuristic Approach to Nurse Rostering
}

\author{
Edmund K. Burke \\ University of Nottingham \\ School of Computer Science \& \\ IT \\ Nottingham NG8 1BB, UK \\ ekb@cs.nott.ac.uk
}

\author{
Patrick De Causmaecker \\ KaHo St.-Lieven \\ Information Technology \\ Gebr. Desmetstraat 1, 9000 \\ Gent, Belgium \\ patdc@kahosl.be
}

\author{
Sanja Petrovic \\ University of Nottingham \\ School of Computer Science \& \\ IT \\ Nottingham NG8 1BB, UK \\ sxp@cs.nott.ac.uk
}

\author{
Greet Vanden Berghe \\ KaHo St.-Lieven \\ Information Technology \\ Gebr. Desmetstraat 1, 9000 \\ Gent, Belgium \\ greetvb@kahosl.be
}

\begin{abstract}
Users of hospital personnel planning software cope with the complex task of translating their needs into several constraints of a very different nature and with differing cost parameters. We present a multi criteria evolutionary approach, which overcomes some of the practical difficulties that personnel schedulers in hospitals often face.
\end{abstract}

\section{INTRODUCTION}

A nurse scheduling problem can be stated as the task of assigning shifts to personnel members who should belong to the proper qualification categories required for the duties that they have to carry out. Over recent years there have been a number of different versions of the problem. Genetic Algorithms $[1,2,13,20]$, tabu search $[10,12]$, simulated annealing [5], variable neighbourhood search [9] and hybrid approaches [7] and their impact on nurse rostering have all been investigated. The assignment is subject to a set of constraints which can vary from hospital regulations to specific personal requests such as holidays and days off. Hard constraints are those which must be satisfied at all costs. We maintain the rule that the coverage constraint (assign the requested number of skilled personnel at any time) can never be violated during the course of the search. Soft constraints are those which are desirable but which may need to be violated in order to create a workable solution.

The nurse rostering definition in this paper is based on the problem description of a commercial personnel planning system called Plane which is aimed at the Belgian hospital market and which is implemented by the authors working with Impakt ${ }^{1}$. A hybrid tabu search algorithm [10], developed for Plane, produces good quality results in a reasonable amount of calculation time. Experiments with memetic, genetic and variable neighbourhood algorithms have led to the methods described in [7, 8, 9]. The approach generates very good results at the expense of calculation time. All of these evolutionary heuristics could be applied to the multi criteria approach discussed in this paper.

The nurse rostering problem is a complex combinatorial problem, which is characterised by multiple goals. Some of the constraints are easier to satisfy than others and that should be taken into consideration within the search algorithm. Very

\footnotetext{
${ }^{1}$ Impakt N.V., Dendermondsesteenweg 42, B-9000 Gent
}

few researchers have worked on multi criteria approaches to nurse timetabling problems. In most approaches, personnel coverage is treated as a goal, unlike in this paper, where it is a hard constraint.

Arthur and Ravindran [3] propose a two phase heuristic for nurse scheduling. The first phase is a zero-one goal programming approach, which aims at minimising the staff size, satisfying the coverage constraints, and meeting the personal requests, while assigning days on and off. The shifts are heuristically assigned to the personnel members in the second phase. Musa and Saxena [16] tackle a rather small size problem in an interactive manner. Users can change the relative weights given to the goals during the scheduling process in order to take special temporal conditions into account. Franz et. al. [14] developed a multi objective integer linear program for health care staff working at different locations. It covers decisions at higher levels than pure short term rostering but the approach does not tackle the problem in as much detail. Ozkarahan [17] defines three basic objective functions for the goal programming approach: maximising the utilisation of full time personnel, minimising over- and understaffing, and minimising several kinds of personnel costs. Compared to our problem, the dimensions of Ozkarahan's approach are considerably smaller. Chen and Yeung [11] combine expert systems and goal programming. Personnel coverage and time related constraints on the personal schedules are the two considered goals and the expert system assigns shifts to individual nurses. In [4], Berrada et. al. apply two different approaches. The first is multi objective mathematical programming, in which slack variables are introduced into the soft constraints with the objective of minimising their values. The second one employs tabu search. In each iteration the most promising move is considered, improving the objective function which is the worst at the time. The results are satisfactory but the program is not really applicable to real-world problems since they only assign days off and working days, and do not specify shifts. The division of the requirements into hard and soft constraints also differs from how it is done in Plane. Moreover, the problem dimensions are much smaller than those addressed in this paper and it is therefore not possible to compare the results. Jaszkiewicz [15] applies simulated annealing in a multi objective approach to generate a set of good quality schedules for problems with few constraints. In an interactive phase afterwards, a hospital scheduler evaluates the obtained results. 
In this paper we present a multi criteria approach within the existing software for nurse scheduling in which criteria are used to evaluate the quality of schedules with respect to violations of constraints. Weights of the criteria reflect the importance of constraints and they are set by the scheduler. Similar approaches have been developed for timetabling problems by Burke et. al. [6] and Paechter et. al. [18]. Although the main concept for the distance measure is the same, the high number of constraints in the nurse rostering approach makes it inconvenient to rely upon the user's experience for setting the targets (as in [18]).

The paper is organised as follows. We explain the major concepts of the cost function approach for Plane in Section II. Some problems for the user of the personnel planning software are also outlined. Section III elaborates on the developed multi criteria method in order to tackle these particular drawbacks of the personnel scheduling problem. We carried out a series of tests and the results are presented in Section IV. In Section V, some conclusions on the new multi criteria approach for the personnel planning problem are drawn.

\section{COST FUNCTION GUIDED APPROACH}

The development of Plane started in 1993 but the system has not stopped evolving as new users appeared with different demands and planning habits. The program currently in use is a very complex system, which can be fine-tuned by the hospital planners in order to meet their requirements.

It has been adapted to an increasing number of user defined constraints, in order to meet the high requirements of all the different hospital wards. The system is very user friendly and provides modifiable functionalities to all customers. However, the growing number of constraints renders the task of assigning cost parameters to constraints increasingly difficult. Also, it is rather artificial to unify constraints with completely different characteristics, such as overtime, weekend work, replacing people with a different qualification class, etc.

In $[7,10]$, the cost function per personal schedule was defined as a linear combination of the violations of the constraints [8]. For evaluating the entire schedule, the cost function values per personnel member are summed. Compared to similar problems described in the literature [2, 12], Plane offers plenty of possibilities for the schedulers to modify the evaluation function by defining different flexible work regulations and by setting the cost parameters related to the soft constraints. The latter, however, sometimes requires more insight into the search heuristics than the hospital planners can be reasonably expected to have.

Plane copes with extremely tough constraints. It aims at producing a satisfiable schedule even when violations of the soft constraints are unavoidable. In order to better meet the customers' expectations, we developed several hybridisations for the algorithms. Some were inspired by manual planning techniques that we observed in the real world (solving weekend constraints, for example), others originated from attempts to fine tune the algorithms under certain circumstances. Some soft constraints conflict with each other during the planning process. They can never be solved at the same time.

The procedure for constructing an acceptable schedule consists of several steps. Initially, a feasible schedule, violating none of the hard constraints, is constructed. After the initial step (see [10] for more details), Plane makes use of metaheuristics to reduce the value of the cost function.

The main ideas behind the algorithms consist of moving shifts from one personnel member to another (provided the person is qualified to do the job).

\section{MULTI CRITERIA APPROACH}

\section{A. Soft Constraints}

In this section, we explain how nurse scheduling problems can be modelled as multi criteria problems. The criteria measure the number of violations of soft constraints. These violations are measured in different units: hours, shifts, days, weekends, and their combinations. A multi criteria approach enables constraints of different nature, expressed in different units, to be treated simultaneously. The soft constraints which are enumerated in this section can take different values for different personnel members in the ward. For details about work regulations and constraint values, we refer to [8]. We will briefly describe the criteria groups based on the units in which they are expressed. For a detailed description of the soft constraints that occur in practice see [21].

Hours: The violation of this particular set of constraints can be measured in terms of hours. Examples of such constraints are: - Minimum time between two assignments

- Minimum/Maximum number of hours worked

Shifts: In order to calculate the violation of constraints belonging to this group, we count assignments of shift types to the personnel members. Depending on the constraint values, some sequences or occurances will lead to violations. This group contains constraints concerning a minimum or maximum total number or sequence of shifts. Also belonging to this group are constraints which require people to work together, constraints on replacing people with a different qualification, etc. Some of these constraints are:

- Maximum number of assignments

- Maximum number of assignments per day of the week

- Maximum number of assignments for each shift type

- Maximum number of a shift type per week

- Maximum number of assignments on bank holidays

- Number of consecutive shift types

- Restriction on the succession of shift types

- Shifts off

- Requested assignments

- People who should/should not work together

- Allow work for an alternative skill category

Days: These constraints are independent from the actual shift types or number of hours scheduled. This constraint category needs information whether or not a person works on a certain 
day. Violations of the minimum or maximum number of days and on consecutive days are expressed (as might be expected) as a number of days.

- Minimum/Maximum number of consecutive days

- Minimum/Maximum number of consecutive free days

- Days off

- Assign 2 free days after night shifts

Weekends: The weekend constraint group is treated separately because weekends seem to attract more attention in real-world examples than other constraints. Violations are expressed as a number of weekends in the following constraints:

- Assign complete weekends

- Assign identical shift types during the weekend

- Maximum number of consecutive working weekends

- Maximum number of weekends in a period of 4 weeks

Miscellaneous: This set of constraints actually covers several of the constraint groups described above. They represent very complex requirements for personnel schedules and require a calculation of hours, shifts, days, etc for the evaluation. Examples are:

- Patterns (enabling specific cyclic constraints)

- Counters evaluating certain assignments over longer periods than the planning period, e.g. in order to balance the workload among colleagues

\section{B. Search Space}

In this section we introduce the search space which is used for the proposed multi criteria approach. We use compromise programming, which is based on the concept of the distance from an ideal solution [22]. Each solution for the problem (a schedule) is represented as a point in a criteria space whose dimension is equal to the number of criteria. Two points in the criteria space have a special meaning: the ideal point and the anti-ideal point. In the ideal point, all criteria take their best value. Very often no solution corresponding to this ideal point exists. We also define an anti-ideal point, represented by the worst value for all the criteria.

In order to tackle all the criteria in dimensionless units, the criteria space is mapped to the preference space [19]. The quality of a solution is expressed in terms of the number of violations of a constraint. Larger co-ordinates indicate worse solutions. For each criterion, the best value is thus mapped to 0 and the worst value is mapped to $w_{c}$, where $w_{c}$ denotes the relative importance (weight) of the criterion c. Obviously, the ideal point is mapped to a point in the preference space, whose co-ordinates are all equal to 0 , while the anti-ideal point is mapped to a point whose co-ordinates are all equal to the weights of the criteria. In this approach the quality of a schedule will be measured by its distance from the ideal point in the preference space. A smaller distance indicates a higher quality.

In order to define a mapping from the criteria space of the nurse rostering problem onto a preference space, a best and worst value are calculated for each criterion (here soft con- straint). We call all the schedules which do not violate a particular constraint ideal schedules with respect to that constraint. These ideal schedules are mapped to points with coordinate 0 for the criterion corresponding to the constraint. An example of a schedule which is ideal in terms of the maximum number of hours worked is a solution in which the constraint on overtime is not violated in any of the personal schedules. For the constraint concerning the minimum number of consecutive days, it suffices to have no shorter sequence of working days in any of the personal schedules, to consider the solution ideal. It is clear that the best value lies in an infeasible part of the solution space for many real-world problems.

The calculation of the worst values of the criteria is more complex. We will illustrate the idea by using a number of examples. Consider the constraints with hourly measures. An estimation of the worst case in terms of overtime is a schedule in which people have to work day and night, without any break during the entire planning period. In this situation, both overtime and the minimum time required between shifts will be violated to the highest extent. In order to violate the undertime constraint to the highest extent, we consider for all the personnel members, a schedule without any assignment.

For the constraints from the shift category which limit the number of assignments, the procedure is analogous to that for the hourly constraints. The consecutiveness constraints, however, require a more careful approach. In order to violate the constraint on the number of consecutive shift types to the highest extent, we imagine a schedule with as many forbidden sequences as possible. The highest number of forbidden sequences is obtained by alternating the smallest forbidden sequence of assigned shifts and a free day. The constraint on complete weekends can be violated most by a schedule in which all Saturdays are free and all Sundays are assigned (or the other way round), for all the personnel members.

Some constraints take the previous planning period into account. If the previous planning period ends with a scheduled day, we will start the worst schedule for 'Minimum number of consecutive free days' with a free day, and vice versa.

For some criteria in which more than one person is involved, we combined full and empty schedules to simulate the worst case. Table I gives an overview of the schedule types which represent the anti-ideal points for each of the criteria. 'Full' and 'empty' schedules are those in which all the possible shifts are assigned and those in which no assignment is made during the planning period.

\section{An Algorithm for Multi Criteria Search}

1) Search Algorithms Our aim is to use the previously developed meta-heuristics for Plane in a new multi criteria environment. Instead of a cost function summing the violations of all the constraints, the driving force is now a minimisation of the distance from a solution to the ideal point in the preference space.

The tests performed for this paper make use of the hybrid tabu search algorithm described in [10]. After an initialisa- 
TABLE I: SCHEDULES REPRESENTING THE WORST CASES OF THE CORRESPONDING CONSTRAINTS

\begin{tabular}{ll}
\hline \hline Constraint & Schedule Characteristics \\
\hline Minimum time between two assignments & Full \\
Maximum number of hours worked & Full \\
$\begin{array}{l}\text { Maximum number of assignments } \\
\text { Maximum number of assignments per day of the }\end{array}$ & Full \\
week & Full \\
Maximum number of assignments for each shift & Full \\
type & \\
Maximum number of a shift type per week & Full \\
Maximum number of working weekends in & Full \\
weeks & \\
Shifts off & Full \\
Maximum number of consecutive days & Full \\
Maximum number of consecutive weekends & Full \\
Maximum number of assignments on bank hol- & Full \\
idays & \\
Days off & Full \\
\hline People who should work together & 1 Full and 1 Empty schedule \\
People who should not work together & Full schedule for both people \\
Counters & Full for this one, empty schedule \\
& for all the other people \\
\hline Number of consecutive shift types & $\begin{array}{l}\text { Alternating the smallest forbid- } \\
\text { den sequence and free days }\end{array}$ \\
Restriction on the succession of shift types & Repeated forbidden sequences \\
Minimum number of hours worked & Empty \\
Requested assignments & Empty \\
Maximum number of consecutive free days & Empty \\
\hline Minimum number of consecutive days & $\begin{array}{l}\text { Alternating scheduled and free } \\
\text { days }\end{array}$ \\
Minimum number of consecutive free days & $\begin{array}{l}\text { Alternating scheduled and free } \\
\text { days }\end{array}$ \\
Assign two free days after night shifts & $\begin{array}{l}\text { Alternating Night Shifts and } \\
\text { empty days }\end{array}$ \\
Assign complete weekends & Every Saturday planned, every \\
& Sunday empty \\
Assign identical shift types during the weekend & $\begin{array}{l}\text { A shift type every Saturday a dif- } \\
\text { ferent shift type every Sunday }\end{array}$ \\
\hline Patterns & Opposite of the pattern \\
\hline &
\end{tabular}

tion phase, which basically consists of assigning all the requested shifts to qualified people randomly, the tabu search algorithm is applied. The algorithm never violates any hard constraints during the calculations. It moves shifts from one person to another on the same day, while respecting the required skill categories. Previous research shows that the algorithm can be improved considerably by adding some hybridisations. The algorithm used to demonstrate this multi criteria approach makes use of two hybridisations: sorting out full weekend work and improving the schedule of the person with the worst result. For more details see [10].

In the cost function approach, the program enables users to set a cost parameter for every constraint. The value of the cost function is the weighted sum of the violations of soft constraints. In an attempt to find a good schedule the cost parameters from the previous approach (Section II and $[7,10]$ ) were copied into the weights of the multi criteria approach. Section IV also presents results with other weight values.

2) Quality of the Schedule Each personal schedule is considered separately by measuring its distance from the ideal point in the preference space. The goal for the search algorithm is to minimise the sum of the distances for $\mathrm{N}$ people in the schedule. We use as a distance measure a family of $L_{p}$ metrics, which gives a wide range of geometric measures for
FIGURE I: MAPPING FROM THE CRITERIA SPACE INTO THE PREFERENCE SPACE FOR PERSON n's SCHEDULE

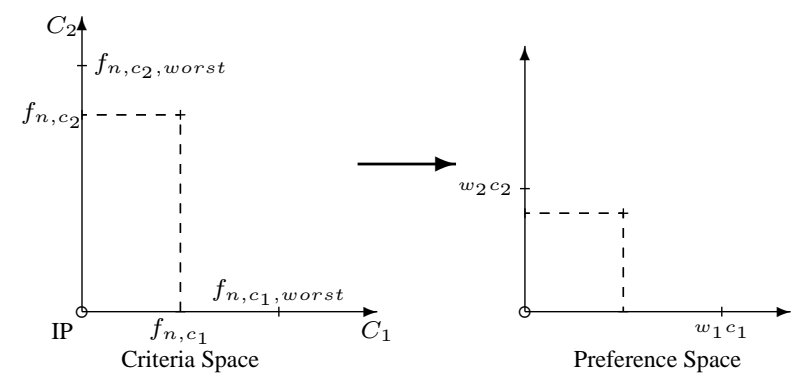

different values of $\mathrm{p}$. A distance from a personal schedule $S_{n}$ for person $\mathrm{n}$ to the ideal point is denoted by $L_{p}(n)$ (Definition 1) where $S_{n}$ has co-ordinates $w_{c} s_{n, c}, \mathrm{n}=1 \ldots \mathrm{N}, \mathrm{c}=1 \ldots \mathrm{C}$ ( $\mathrm{C}$ is a number of objectives) in the preference space.

Definition $1 L_{p}(n)=\left(\sum_{c}\left[w_{c} s_{n, c}\right]^{p}\right)^{1 / p} ; 1 \leq p \leq \infty$ In the definition, $s_{n, c}$ is $\frac{f_{n, c}}{f_{n, c, w o r s t}}$, where $f_{n, c}$ is the value of criterion $\mathrm{c}$ in the schedule of person $\mathrm{n}$ and $f_{n, c, \text { worst }}$ is the value of the criterion $\mathrm{c}$ for the worst possible schedule for $\mathrm{n}$. Smaller values of $\mathrm{p}$ allow for compensation among criteria values, i.e. high satisfaction for one constraint can counterbalance low satisfaction for another one. If the distance measure is $\infty$, the distance $L_{p}(n)$ defined in Definition 1 is the value of the largest co-ordinate in the preference space. Only the most violated constraint will contribute to the value.

Definition $2 L_{\infty}(n)=\max _{c}\left[w_{c} s_{n, c}\right]$

The distance of the entire schedule $S$ is the sum, for all the N nurses in the ward, of the personal schedules (as denoted by Definition 3).

Definition $3 L_{p}(S)=\sum_{n=1}^{N} L_{p}(n)$

The mapping of the criteria space into the preference space, (with $\mathrm{C}=2$ ), is depicted in Fig. I. Fig. II presents the multi criteria search algorithm.

\section{EXPERIMENTS}

\section{A. Test Problems}

The method presented in this paper has been tested on real world data. In order to carry out preliminary experiments, it was our deliberate choice to take a rather simple example. In comparison with other real-world nurse rostering problems, it has a smaller number of constraints and thus a smaller search

\section{FIGURE II: PSEUDO CODE FOR MULTI CRITERIA SEARCH}

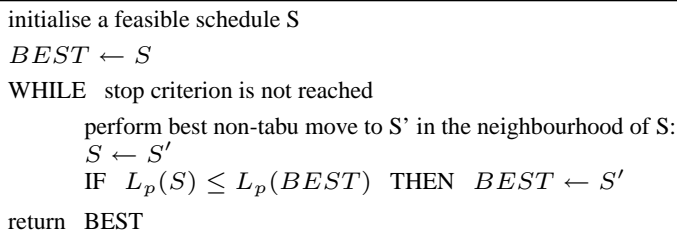


TABLE II: RESULTS FOR DATASET 1

\begin{tabular}{|c|c|c|c|c|}
\hline \multicolumn{3}{|l|}{ Multi Criteria } & \multicolumn{2}{|l|}{ Violations } \\
\hline Weights & $\mathrm{p}$ & $L_{p}(S)$ & Constraints & $\#$ \\
\hline all weights 1 & 2 & 14 & patterns & 8 \\
\hline $\begin{array}{l}\text { patterns } 2 \\
\text { other weights } 1\end{array}$ & & 56 & patterns & 8 \\
\hline $\begin{array}{l}\text { patterns } 3 \\
\text { other weights } 1\end{array}$ & & 117 & patterns & 7 \\
\hline $\begin{array}{l}\text { patterns } 4 \\
\text { other weights } 1\end{array}$ & & 336 & patterns & 7 \\
\hline $\begin{array}{l}\text { patterns } 5 \\
\text { other weights } 1\end{array}$ & & 525 & patterns & 7 \\
\hline $\begin{array}{l}\text { patterns } 10 \\
\text { other weights } 1\end{array}$ & & 1300 & patterns & 7 \\
\hline patterns 50 & & 22707 & \# weekends in 4 weeks & 1 \\
\hline other weights 1 & & & $\begin{array}{l}\text { min. consecutive days } \\
\text { patterns }\end{array}$ & $\begin{array}{l}1 \\
5\end{array}$ \\
\hline cost parameters & & 225 & patterns & 5 \\
\hline Cost Function & & & \multicolumn{2}{|l|}{ Violations } \\
\hline Cost Parameters & & Cost & Constraints & $\#$ \\
\hline & & 250 & patterns & 5 \\
\hline
\end{tabular}

space. It enabled us to carry out experiments with several approaches in a limited amount of calculation time. After promising early results, this approach will be tested on more complex real world data.

In Dataset 1, the personnel requirements consist of 4 different shift types, and a planning period of 4 weeks is considered. The ward employs 9 people belonging to 2 different skill categories; one head nurse and 8 regular nurses. The head nurse has a special work regulation (called Head Nurse) which allows day shifts only and no weekend work. Among the regular nurses, five of them have a Full Time work regulation, one has a personalised full time regulation (forbidding late and night shifts on Tuesday) and the other two are Half Time nurses. This particular problem has some extra difficulties, affecting the worst case values of some personal constraints. One full time nurse is hired by another ward during the first two weeks of the planning period. Another full time nurse leaves the ward after two weeks. Furthermore, one half time and one full time nurse swap work regulations after the first week of the planning period. The data in Dataset 2 represent the same ward as in Dataset 1 but the planning period is different. It also involves different qualifications and work regulations for some personnel members, another position in the cyclic patterns, different personal requests for days off, different bank holidays, and a slightly different personnel demand on some days.

The tests have been carried out with distance measure $p=1,2$, and $\infty$ in the preference space.

\section{B. Discussion}

Table II presents the test results for Dataset 1. In Table III some test results are displayed for Dataset 2. Experiments have been carried out with different values for the weights. In the last two columns, the violated constraints are presented together with the number of times the constraint is violated. The last column sums the number of violations when they occur in different personal schedules. An ideal schedule does not vio-
TABLE III: RESULTS FOR DATASET 2

\begin{tabular}{|c|c|c|c|c|}
\hline \multicolumn{3}{|l|}{ Multi Criteria } & \multicolumn{2}{|l|}{ Violations } \\
\hline Weights & $\mathrm{p}$ & $L_{p}(S)$ & Constraints & $\#$ \\
\hline all weights 1 & 1 & 19 & $\begin{array}{l}\text { min. consecutive days } \\
\text { patterns }\end{array}$ & $\begin{array}{l}1 \\
9\end{array}$ \\
\hline patterns 10 & & 38 & hours & 3 \\
\hline other weights 1 & & & $\begin{array}{l}\text { min. consecutive days } \\
\text { patterns }\end{array}$ & $\begin{array}{l}2 \\
2\end{array}$ \\
\hline patterns 50 & & 8 & hours & 1 \\
\hline other weights 1 & & & min. consecutive days & 7 \\
\hline patterns 50 & & 312 & hours & 6 \\
\hline $\begin{array}{l}\text { min. cons. days } 10 \\
\text { other weights } 1\end{array}$ & & & patterns & 12 \\
\hline all weights 1 & 2 & 72 & $\begin{array}{l}\text { max. consecutive days } \\
\text { patterns }\end{array}$ & $\begin{array}{l}3 \\
7\end{array}$ \\
\hline patterns 2 & & 114 & hours & 2 \\
\hline other weights 1 & & & patterns & 8 \\
\hline patterns 5 & & & hours & 2 \\
\hline other weights 1 & & & patterns & 6 \\
\hline patterns 10 & & & min. consecutive days & 2 \\
\hline other weights 1 & & & identical weekends & 2 \\
\hline & & & patterns & 2 \\
\hline cost parameters & & 0 & & \\
\hline \multirow[t]{8}{*}{ all weights 1} & $\infty$ & 125 & hours & 13 \\
\hline & & & max. assignments & 1 \\
\hline & & & \# weekends in 4 weeks & 3 \\
\hline & & & day off & 3 \\
\hline & & & min. consecutive days & 5 \\
\hline & & & complete weekends & 1 \\
\hline & & & identical weekends & 1 \\
\hline & & & patterns & 9 \\
\hline patterns 10 & & 777 & hours & 74 \\
\hline \multirow[t]{7}{*}{ other weights 1} & & & max. assignments & 6 \\
\hline & & & \# weekends in 4 weeks & 3 \\
\hline & & & day off & 4 \\
\hline & & & min. consecutive days & 13 \\
\hline & & & complete weekends & 2 \\
\hline & & & identical weekends & 6 \\
\hline & & & patterns & 14 \\
\hline \multirow[t]{11}{*}{ cost parameters } & & 3642 & hours & 192 \\
\hline & & & max. assignments & 18 \\
\hline & & & max. consecutive days & 6 \\
\hline & & & \# weekends & \\
\hline & & & in 4 weeks & 1 \\
\hline & & & min. time between & 78 \\
\hline & & & day off & 5 \\
\hline & & & min. consecutive days & 6 \\
\hline & & & complete weekends & 1 \\
\hline & & & identical weekends & 7 \\
\hline & & & patterns & 14 \\
\hline \multicolumn{3}{|l|}{ Cost Function } & Violations & \\
\hline \multirow{2}{*}{ Cost Parameters } & & Cost & Constraints & \# \\
\hline & & 7 & min. consecutive days & 7 \\
\hline
\end{tabular}

late any soft constraint. Both test examples demonstrate that increasing the relative importance of a particular constraint often results in a schedule with less violations of that constraint. This does not generally hold because of the local optima. The best solution for Dataset 1 violates the 'pattern' constraint 5 times. We know, after investigating the problem data thoroughly, that there is no solution which corresponds to the ideal point. When assigning a moderate weight to the pattern constraint, it is violated more often. Since the data of the cost function approach is available anyway, we experimented with copying the cost parameters into the weight factors of the corresponding constraints. It is not irrelevant to assume a correspondance between the cost parameters set by the users and the importance of the corresponding constraints. This option for the weights led to the best results for the multi criteria approach.

The results from the Dataset 2 example clearly show that a 
lower value of the distance measure $p$ enables compensations between constraints. With higher values of $p$, the algorithm tends to generate schedules with a reduced penalty of the most violated constraint because its contribution to the value of the cost function is very high. The results with distance measure $\infty$ are not promising at all. The first experiment was carried out with all weights equal to 1 . It is only the pattern constraint which determines the value of the distance, although many other constraints are also violated. Increasing the weight for the patterns seems the most obvious thing to do, but as presented on the next row in Table III, the results are even worse. For some people, the violation of that particular constraint has decreased at the expense of other constraints. In the schedule of some personnel members, when other constraints are responsible for the distance, the violation on patterns has even increased. Copying the cost parameters into the weights gives the worst results of all, whereas this was the best option for the $\mathrm{p}=2$ distance measures.

Increasing the weights for the most violated constraint almost always leads to better quality solutions in which less constraints are violated. Also for this test example, copying the cost parameters into the weights is the best option. It is not possible to compare the results obtained using the cost function and the multi criteria approach because they are based on different evaluation functions. In fact, the comparison of these two approaches based on numbers of all the constraint violations in the obtained schedules is a multi criteria problem in itself. However, in one particular case, we can see that the multi criteria approach outperforms the single cost function approach. This happened in the Dataset 2 example when the multi criteria approach gave 0 violations of all of the constraints while the cost function approach resulted in 7 violations on 'Minimum consecutive days'.

\section{CONCLUSIONS}

The new multi criteria meta-heuristic approach presented in this paper overcomes some practical difficulties for automated nurse rostering, which is a multi criteria problem by nature. It enables handling dissimilar constraints in a better way than a single cost function approach does by taking the ranges of possible criteria into consideration. The developed multi criteria approach, incorporated in a meta-heuristic, allows the scheduler to control the compensation of constraints. Tests on real world data have shown that previously developed metaheuristic algorithms perform very well using the new multi criteria approach. In many cases, the resulting personnel rosters outperform those found by the cost function driven approach.

Our future work will include investigations on meta-heuristics which modify the weights of criteria in order to escape from local optima. We will take into account the fact that some constraints are easier to satisfy and combine that with the weights of the criteria. In general, the multi criteria approach presented in this paper has the potential to accommodate the every day practice of hospital schedulers much more closely than the cost function approach.

\section{References}

[1] J. Ahmad, M. Yamamoto, A. Ohuchi: Evolutionary Algorithms for Nurse Scheduling Problem, Proceedings of CEC00, San Diego, 2000 , IEEE Press, 196-203

[2] U. Aickelin, K. Dowsland: Exploiting problem structure in a genetic algorithm approach to a nurse rostering problem, Journal of Scheduling, Vol. 3, No. 3, 2000, 139-153

[3] J. Arthur, A. Ravindran: A Multiple Objective Nurse Scheduling Model, AIIE Transactions, Vol. 13, No. 1, 1981, 55-60

[4] I. Berrada, J. Sirland, P. Michelon: A Multi-Objective Approach to Nurse Scheduling with both Hard and Soft Constraints, SocioEconomic Planning Science 30, 1996, 183-193

[5] M.J. Brusco, L.W. Jacobs: Cost analysis of alternative formulations for personnel scheduling in continuously operating organisations, European Journal of Operational Research 86, 1995, 249-261

[6] E.K. Burke, Y. Bykov, S. Petrovic: A Multicriteria Approach to Examination Timetabling, E.K. Burke, W. Erben (Eds.): Practice and Theory of Automated Timetabling, Konstanz, Springer, 2000, 118-131

[7] E.K. Burke, P. Cowling, P. De Causmaecker, G. Vanden Berghe: A Memetic Approach to the Nurse Rostering Problem, App. Intell. special issue on Sim. Ev. and Learning, Vol. 15, No. 3, 2001, 199-214

[8] E.K. Burke, P. De Causmaecker, S. Petrovic, G. Vanden Berghe: Fitness Evaluation for Nurse Scheduling Problems, Proceedings of CEC2001, Seoul, 2001, IEEE Press, 1139-1146

[9] E.K. Burke, P. De Causmaecker, S. Petrovic, G. Vanden Berghe: Variable Neighbourhood Search for Nurse Rostering Problems, submitted to MIC, 2001

[10] E.K. Burke, P. De Causmaecker, G. Vanden Berghe: A Hybrid Tabu Search Algorithm for the Nurse Rostering Problem, X. Yao et al. (Eds.): SEAL'98, LNCS 1585, 1999, 187-194

[11] J.-G. Chen, T. Yeung: Hybrid Expert System Approach to Nurse Scheduling, Computers in Nursing, 1993, 183-192

[12] K. Dowsland: Nurse scheduling with Tabu Search and Strategic Oscillation. European Journal of Operations Research (106), 1998, 393-407

[13] F. Easton, N. Mansour: A Distributed Genetic Algorithm for Employee Staffing and Scheduling Problems, Conference on Genetic Algorithms, San Mateo, 1993, 360-367

[14] L.S. Franz, H.M. Baker, G.K. Leong, T.R. Rakes: Mathematical model for scheduling and staffing multiclinic health regions, European Journal of Operational Research, Vol. 41, No. 3, 1989, 277-289

[15] A. Jaszkiewicz: A metaheuristic approach to multiple objective nurse scheduling, Foundations of Computing and Decision Sciences, Vol. 22, No. 3, 1997, 169-184

[16] A. Musa, U. Saxena: Scheduling Nurses Using Goal-Programming Techniques, IEEE, 1984, 216-221

[17] I. Ozkarahan: An Integrated Nurse Scheduling Model, Journal of the Society for Health Systems, Vol. 3, No. 2, 1991, 79-101

[18] B. Paechter, R.C. Rankin, A. Cumming, T.C. Fogarty: Timetabling the Classes of an Entire University with an Evolutionary Algorithm, PPSN V, Springer, LNCS 1498, 1998

[19] Petrovic, S., Petrovic R.: Eco-EcoDispatch: DSS for multicriteria loading of thermal power generators, Journal of Decision Systems 4 (4), 1995, 279-295

[20] J. Tanomaru: Staff Scheduling by a Genetic Algorithm with Heuristic Operators, Proceedings of CEC95, 1995, 456-461

[21] G. Vanden Berghe: Soft constraints in the Nurse Rostering Problem, http://www.cs.nott.ac.uk/ gvb/constraints.ps, 2001

[22] M. Zeleny: A Concept of Compromise Solutions and the Method of the Displaced Ideal, Computers and Oper. Res., Vol. 1, 1974, 479-496 